\title{
Cerebral mechanisms operating in the presence and absence of inflammatory pain
}

\author{
Anthony K P Jones, Stuart W G Derbyshire
}

During episodes of inflammatory arthritic pain, the brain has the task of integrating new information from receptors of muscle, tendon, and skin afferents with nociceptive information from the joint capsule and, possibly, periarticular bone. This integration will incorporate motivational, affective, discriminative, attentional, and motor responses that may result in changes of behaviour and modification of central nervous system (CNS) processing of pain. It is as yet unclear what the nature of this CNS modification may be, and suggested influences on pain related affective-motor responses or the inflammatory process itself remain largely speculative.

It is likely to be some time before we begin to have a proper understanding of the complex integrated processes that operate during arthritic pain and other chronic pains. However, we are now able to measure aspects of those processes in terms of measurement of behavioural and cerebral responses to pain. These measurements are providing early clues to the possible underlying CNS mechanisms and their relation to changes in affectivemotivational behaviour. This paper will review the normal brain mechanisms of the conscious appreciation of pain, together with working hypotheses for how these mechanisms are altered during inflammatory and other types of clinical pain syndromes.

\section{Normal mechanisms: anatomy and physiology}

It is perhaps surprising that there should still be debate about which areas of the brain might be involved in the processing of such an important and commonplace sensation as pain. This has come about largely because of the understanding of pain as an experience involving sensory, cognitive-evaluative, and affectivemotivational components ${ }^{1}$ which defies simple explanations involving pain centres ${ }^{1}$ and specific pathways. ${ }^{2}$

Whatever areas of the brain are involved in pain processing, integration of the different components must be accommodated. This implies that there is unlikely to be any final common area of the brain that processes pain. Indeed, it is perhaps the search for a single dominant 'pain centre' that has done more to impair our understanding of the functional anatomy of pain than anything else. Parallel processing of the conscious appreciation of primary sensations such as vision and touch ${ }^{3}$ are now well documented, and it would be surprising if such parallel processing did not also exist for non-primary sensations such as pain. There is now growing evidence that pain is processed by a network or 'matrix' of structures in the brain (fig 1), some components of which could be considered to comprise parallel but integrated processing.

NEUROANATOMY OF NOCICEPTION IN ANIMALS Studying pain in animals presents an insoluble problem. Human beings can call out and express their pain in ways that are commonly understandable to other members of their species; unfortunately, there is no equivalent frame of reference when dealing with animals. Increased blood pressure, movements of withdrawal, dilatation of the pupil, increased depth of ventilation, attacking the source of noxious stimulation, and cries may be common to all mammals in the face of seemingly 'painful' stimulation, but all such responses can be elicited after the cerebral cortex has been destroyed, in the probable absence of any subjective experience. Furthermore, the subjective experiences of an animal, if it has any, may be totally different from those of humans. For these reasons, human interpretation of what is observed in another species cannot be by extrapolation from human experience.

This is not to suggest that all 'pain' research in animals is valueless: the common ancestry between humans and animals suggests that some of the underlying biological processes involved in human pain experience are likely to be shared with other animals. A distinction is therefore drawn between 'nociception' and pain-which should not be used interchangeably - the term nociception being used to refer to the biological response of receptors in tissue to potentially damaging stimuli such as intense heat or cold, chemical irritation, or intense pressure. The study of nociceptive pain avoids the fact that pain in man can occur without any evident nociceptor activation, as in peripheral and central deafferentation pain, and may also occur without any evidence of spinothalamic tract activation, as in psychogenic pain.

The spinothalamic tract has been demonstrated to be 'both necessary and sufficient' for nociception in most species studied. ${ }^{4}$ It provides the major direct nociceptive input from the spinal cord to the thalamus. As a consequence, the spinothalamic tract ascending in the anterolateral cord is widely considered as the 'pain pathway'. 
Spinothalamic tracts

In the early 19th century, Brown-Sequard performed studies in which the ventrolateral quadrants of the spinal cord were sectioned in animals and the resulting effects compared with sensory deficits caused by similar lesions in humans. ${ }^{5}$ Sherrington and Laslett ${ }^{6}$ observed 'that the lateral column furnishes the headward path in the spinal cord for nociceptive (algesic) arcs' and 'that this is true for these arcs, whether they are traced from skin, muscle or viscera'. Lesioning of the ventral quadrant in monkeys results in a consistent increase in response thresholds to nociceptive stimuli on the contralateral side below the level of the lesion. ${ }^{78}$ This analgesic response usually recovers one to six months after the lesioning. ${ }^{9}$ The most long lasting analgesic effects were produced by the most extensive lesions including both dorsal and ventral quadrants, indicating that other pathways may also transmit nociceptive information, or that the spinothalamic tract may have important dorsal components. Quantitative studies also have demonstrated an important contribution of the dorsolateral fasciculus to the spinothalamic tract. ${ }^{10}$ The thalamus was injected with agglutinin conjugated horseradish peroxidase (HRP) in the presence and absence of thoracic spinal lesions to preserve either the ipsilateral or contralateral ventral quadrant or dorsolateral fasciculus: $25-30 \%$ of the cells staining for HRP in the lumbar segments were accounted for by retrograde transport of stain down the contralateral dorsolateral tract. These authors also found significant differences in the distributions of cells of origin of these two pathways. The most striking observation was the predominance of laminae I-III of the dorsal horn as the main sites of origin of the dorsolateral funiculus and laminae VII-X as the main sites of origin of the ventral quadrant of the spinothalamic tract. Laminae IV-VI made a variable and often significant contribution, dependent on the species of monkey. An ipsilateral origin was demonstrated for $17 \%$ of both components of the spinothalamic tracts. ${ }^{10}$

The terminations of the spinothalamic tracts in different thalamic nuclei have also been studied using anterograde transport of HRP injected into the lumbar segments in monkeys. ${ }^{11}$ Extensive termination of both components of the spinothalamic tracts was demonstrated in both the lateral and medial thalamic nuclei. The lateral terminations were predominantly to the suprageniculate nucleus, pulvinar oralis, the caudal and oral divisions of the ventral posterior lateral nucleus, the ventral posterior inferior nucleus, and the zona incerta. In the medial thalamus, the main terminations were in the medial dorsal, intralaminar nuclei of the central lateral, central median, and parafascicular nuclei. There was extensive overlap between the regions innervated by the ventral and dorsal quadrants of the spinothalamic tracts. However, there were more extensive projections of the ventral spinothalamic tract to the medial thalamic nuclei. Other anterograde degeneration studies have also shown extensive spinothalamic projections to the midline thalamic nuclei. ${ }^{12}$
The cortical projections of the thalamic nuclear terminations of the dorsal and ventral spinothalamic tracts are not clearly defined in any species. Although the cortical connections of some of the more important nuclei have been described in different species, it cannot be assumed that all these connections are necessarily relevant to the spinothalamic terminations. The following descriptions of cortical projections can only therefore be a pointer to some of the possible functional connections in relation to the spinothalamic tracts.

Both components of the spinothalamic tracts have a heavy projection to the caudal portion of the ventral posterior lateral nucleus of the thalamus. This nucleus, in addition to the ventral posterior inferior and the centrolateral nuclei of the thalamus, have important projections to neurones in the primary somatosensory cortex (SI) which are probably nociceptive. ${ }^{13}$ There are also probable nociceptive projections from thalamic nuclei (ventral posterior lateral, posterior nucleus, and the centrolateral nucleus) to the secondary somatosensory cortex (S2). ${ }^{14}$ Although it has been shown that $\mathrm{S} 1$ contains units that respond to noxious stimuli, ${ }^{15}$ Shi et al ${ }^{13}$ have argued that 'nociception does not seem to be a sensory modality that is prominently represented in either the first or the second somatic sensory area of the parietal cortex. Since the terminals of the [spinothalamic tract] are scattered in small patches, since the cortical projections from the $\mathrm{VPL}_{\mathrm{c}}$ (caudal section of the ventral posterior lateral nucleus) are restricted to small areas, ${ }^{16}$ since $\mathrm{VPL}_{\mathrm{c}}$ cells in a given somatotopic area project to multiple cortical zones and not all $\mathrm{VPL}_{\mathrm{c}}$ cells project to the cortext ${ }^{17} \ldots$.

The pulvinar oralis and the suprageniculate nucleus have been termed the posterior region of the thalamus, and this region receives overlapping terminations from both spinothalamic components. This area in turn projects to the insular cortices in primates ${ }^{18}$ which, because of its connections with the amygdala, has been implicated in the autonomic responses to nociceptive stimuli. A subdivision of the ventral medial nucleus of the thalamus in the ventroposterior part of the lateral thalamus has recently been identified as being a relay nucleus for nociceptive specific information from lamina I of the dorsal horn in primates. ${ }^{19}$ This nucleus also projects to the anterior insula cortex, and may be a source of nociceptive inputs to the insula parallel to those from the medial thalamic nuclei. The cortical projections of the medial thalamic nuclei are described below.

\section{Spinoreticulothalamic tracts}

Studies in the macaque monkey ${ }^{2021}$ have demonstrated that fibres in the anterior part of the anterolateral spinothalamic tract originate in the contralateral posterior horns and end in the gigantocellular part of the medulla and pons, and in the lateral reticular nucleus. Fibres or collaterals from these areas terminate in the medial geniculate body, the posterior group, and the intralaminar nuclei of the thalamus. The fibres of the spinoreticulo- 
thalamic tract may be divided into a caudal and ventral group of fibres: the caudal fibres terminate in the central median, parafascicular, centrolateral, and dorsomedial nuclei of the thalamus, while the ventral group synapses in the subthalamus and the hypothalamus. There is also a connection with the Edinger-Westphal nucleus, which may be the pathway mediating the pupillary dilatation in response to noxious stimuli. Fibres from the ventrolateral spinothalamic tract have a crossed and double crossed projection to a reticular nucleus in the medulla-the subnucleus reticularis dorsalis. This nucleus appears to have nociceptive specific inputs that are totally convergent on units with hemi or whole body receptive fields. Efferent projections are to the parafascicular and ventromedian nuclei of the thalamus. ${ }^{22}{ }^{23}$ Projections via the dorsal accessory olive to motor areas such as motor and premotor cortices and striatum suggest possible involvement in arousal and motor reactions.

Cortical projections of the medial thalamic nuclei: medial $\mathrm{v}$ lateral pain systems

The intralaminar group of nuclei are an important group of medial thalamic nuclei that were originally described as those nuclei that lay within the internal medullary lamina of the thalamus. However, they are now considered to comprise the central medial nucleus located in the midline and the more lateral paracentral and central lateral nuclei, and a third more posterior group that includes the posterior intralaminar, centromedian and parafascicular nuclei. These structures have for many years

MEDIAL

Schema retrieval Attention supervision Motor inhibition

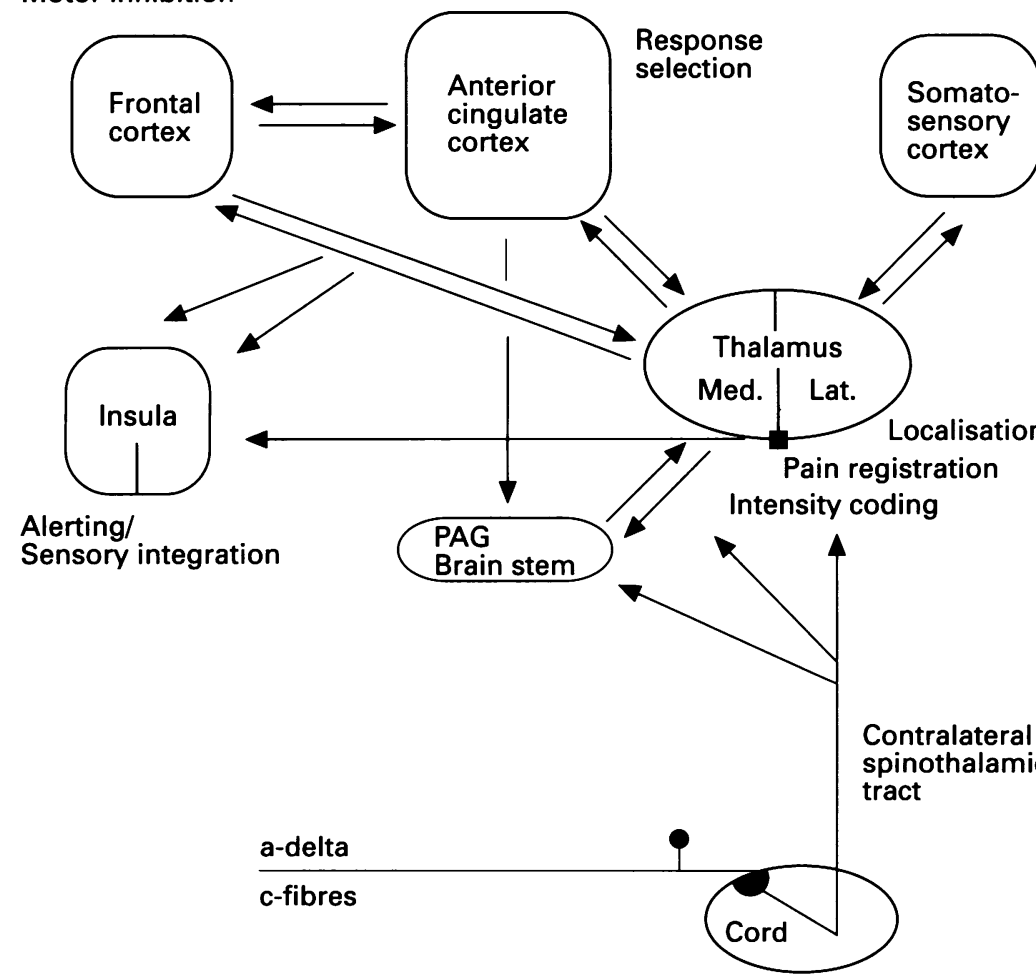

Figure 1 Schematic diagram of some of the main anatomical components of the 'pain matrix', and their possible functional significance. $P A G=$ Periaqueductal grey matter. been considered on anatomical grounds to be a cranial extension of the brain stem reticular formation. ${ }^{24}$ In addition to the mediodorsal nucleus, they have been found to have important connections with both the cingulate ${ }^{25}$ and prefrontal cortex ${ }^{26}$ in the monkey. Cells within these medial thalamic nuclei respond to nociceptive stimuli and often have large and often bilateral receptive fields with a non-somatotopic organisation. ${ }^{22}$ For these reasons, these structures are considered to subserve the so called 'medial pain system', as distinct from the somatotopically arranged projections to the ventral posterior lateral nucleus which are thought to subserve the 'lateral pain system'. ${ }^{26 a}$ Vogt et al considerably extended the concept of the medial pain system by demonstrating projections of the medial thalamic group of nuclei to area 24 of the anterior cingulate cortex. ${ }^{27}{ }^{28}$ The anterior cingulate cortex is an extensive area of the limbic cortex overlying the corpus callosum which is involved in the integration of cognition, affect, and response selection. ${ }^{29}$ There is also evidence for medial thalamic nociceptive projections to the prefrontal cortex. ${ }^{30}$ The descending connections of the anterior cingulate cortex to the medial thalamic nuclei and to the periaqueductal grey in the brain stem suggest that this system may also be involved in the modulation of reflex responses to noxious stimuli. ${ }^{28}$

The lateral pain system is subserved by monosynaptic projections from the dorsal horn and is therefore rapid compared with the polysynaptic medial pain system. This makes it a more likely candidate for processing information about acute pain. Certainly 'first pain', which is that first well defined pricking or stabbing sensation, would require such a relatively rapid system. However, acute pain incorporates both first pain and second pain. The former is important for avoidance of potentially damaging stimuli. 'Second pain', which is that more enduring, often burning and unpleasant, slow component of pain, is likely to be of greater relevance to the experience of most acute clinical pain. Groups of neurones have been identified in the contralateral primary and secondary sensory cortices of unanaesthetised primates capable of responding to noxious stimuli. ${ }^{31}$ These have traditionally been considered to derive from the ventral posterior lateral nucleus of the thalamus, and their presence has reinforced the classical idea that the 'lateral pain system' is the main system for processing acute pain, whereas the medial pain system is the main system processing chronic pain. An alternative hypothesis is that the lateral pain system is dealing primarily with the sensory discriminative aspects of pain, whereas the medial pain system is concerned mainly with the motivational-affective components of pain response. ${ }^{32}$

THE CEREBRAL NETWORK FOR PAIN PROCESSING IN MAN

The cerebral neural substrate mediating painful response to noxious stimuli in man has 
been poorly understood until recently. Studies in man to identify the principal areas of the brain involved in the response to pain have relied predominantly either on stimulating specific areas of the brain in order to relieve $\mathrm{e}^{33}$ or elicit pain known to have inputs from the spinothalamic tracts, ${ }^{34}$ or on observing the effects of brain lesions resulting from an infarct or surgery. ${ }^{35-38}$ These different approaches have provided some very important insights into human pain physiology, but have not identified those areas of the brain germane to the integrated response to painful stimuli in normal individuals. Penfield and Boldrey observed that only 11 of 800 cortical stimulus locations in the primary motor sensory cortex elicited pain when stimulated, ${ }^{39}$ and none of these sites produced severe pain, but mostly a sharp burning pain. This observation has generated considerable debate as to the relative importance of the cerebral cortex to pain sensation in man. In more recent studies in patients undergoing craniotomy for severe intractable pain, pain was elicited by stimulating the primary and secondary sensory cortices and in some cases this has reproduced the type of chronic pain experienced by the subject. ${ }^{40-42}$ It is difficult to know whether these experiences in response to this type of stimulation result from local neuronal excitation or as a result of activation of corticothalamic pathways. Because these studies are difficult to reproduce, it is difficult to assess how relevant these findings are to normal pain physiology or to the majority of patients suffering from different types of chronic pain. Experiments in primates suggest that the cingulate cortex plays an important part in conditioning responses to painful stimuli, ${ }^{43}$ while it has been observed that removal of the frontal or cingulate cortex in patients with intractable pain may ameliorate its unpleasant emotional component without necessarily abolishing the pain itself ${ }^{37}$ or the perception of acute nociceptive stimuli. ${ }^{35}{ }^{41} \mathrm{It}$ is interesting that Foltz and White ${ }^{37}$ had also observed that, after frontal cingulumotomy, no patient who had been addicted to opioids before surgery required opioids after the operation. Of the 14 patients who had been addicted, nine had no withdrawal symptoms and only five had mild withdrawal symptoms, suggesting that this operation may attenuate opioid withdrawal symptoms. The significance of these structures to the integrated normal human pain response has been unknown until recently because of the lack of non-invasive techniques for observing such responses in specific brain regions.

Electrophysiological techniques have been extensively reviewed by Chen. ${ }^{44}$ Much current information regarding the pain network is the result of recent studies using functional imaging techniques such as positron emission tomography (PET) and functional magnetic resonance imaging (fMRI). These techniques, which offer exciting possibilities for theoretical advancement, enable imaging of events in the grey matter of the brain, giving a 'snapshot' of the actual function of the brain. They are noninvasive techniques that have recently been developed to quantitate changes in blood flow in man as an index of neuronal activity in response to physiological stimuli. ${ }^{45}$ The underlying assumption in applying these techniques is that there is a close relationship between blood flow and neuronal function. There is good evidence from animal studies that there is such a relationship, and that changes in blood flow are closely related to neuronal energy metabolism. ${ }^{46}$ These methods therefore provide the exciting possibility of defining functional neuroanatomical substrates of different internally or externally generated physiological stimuli, and have been extended to studies of response to non-painful and painful thermal stimuli in normal volunteers, to determine which areas of the brain respond specifically to acutely painful thermal stimuli applied to the back of the right hand, compared with nonpainful thermal stimuli applied to the same spot. Experiments were therefore designed specifically to exclude the temporal and spatial localisation components of the response. The hypothesis to be tested was that pain is a predominantly emotional experience and therefore substantial specific responses to pain are more likely to be seen in the cortical and subcortical structures relating to the 'medial pain system'.

An initial group of experiments examined the non-sensory discriminative components of the cerebral response to an acute painful stimulus. A series of painful or non-painful phasic stimuli were given for two minutes while regional cerebral blood flow was measured. The effect of non-painful heat was subtracted from the effect of painful heat so that the temporal and spatial discriminatory components of the pain were controlled, leaving only the motivationalaffective and cognitive components of the pain response. The subjects did not move during the delivery of the stimuli and were therefore possibly suppressing the desire to withdraw the hand. The results in a number of groups of pain free volunteers have shown that there is a unique network of structures activated by the non-discriminatory components of pain which is not activated during other cognitive, motor, or sensory tasks.

The non-discriminatory components of an acute thermal pain stimulus activate predominantly the contralateral thalamus, anterior insula, lentiform nucleus and anterior cingulate cortex, ipsilateral dorsolateral prefrontal cortex (Brodman area 9 and 10), and inferior parietal cortex (areas 39 and 40) (table). ${ }^{47-50}$ Subsignificant activations have also been seen

Summary of the areas of significant $(p<0.05)$ increases in regional cerebral blood flow ( $\mathrm{CCBF}$ ) in response to pain for each of the studies indicated

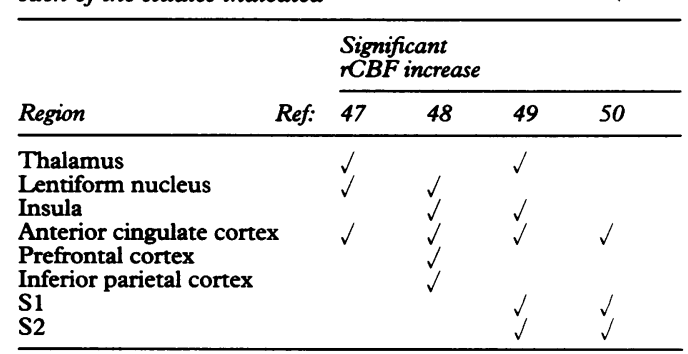

S1, S2 = Primary and secondary somatosensory cortex 
in the region of the periaqueductal grey in the brain stem. A similar pattern of activation has been observed in normal volunteers subjected to a tonic heat stimulus to the back of the right hand. ${ }^{48}$ Confirmation of these areas being activated by acute pain has come from a number of different laboratories including groups from Ann Arbor, ${ }^{49}$ Montreal, ${ }^{50}$ Rome,,${ }^{51}$ and Stockholm (Hsieh JC, et al; submitted for publication). However, there is also some important variability. In addition to the midcingulate area previously described, a recent study has demonstrated an additional highly significant area at the perigenual cingulate. ${ }^{52}$ Studies disagree as to the laterality of cingulate response, some indicating bilateral responses; there are also reports of bilateral thalamic activation. ${ }^{52}$ The possibility arises that there may be a bilateral component to thalamic, insula, and basal ganglia processing, though the contralateral component may well be quantitatively more important. Nociceptive response in units in area 24 of the anterior cingulate often exhibit whole body receptive fields. Some bilaterality of response might therefore be expected in this region. Although PET studies suggest that acute pain responses are mainly contralateral, chronic neurogenic pain appears to be processed ipsilaterally. ${ }^{53}$ It should be remembered that the effective spatial resolution of activation studies is about $16 \mathrm{~mm}$ and therefore there always remains some uncertainty about the distinction between structures either side of the midline. However, fMRI studies also suggest that there is bilateral anterior cingulate activation in response to pain. ${ }^{54}$

There is also unexpected variability of responses in the somatosensory cortex. The Manchester/Hammersmith group have been unable to elicit consistent responses in $\mathrm{S} 1$, finding both increases and decreases in flow in response to pain. Apkarian et al have found decreases in flow in response to tonic pain in S1/S2. ${ }^{55}$ Both the Montreal and Ann Arbor groups have documented responses in this area of cortex, ${ }^{49}{ }^{50}$ and contralateral responses in S2 to phasic pain ${ }^{49}$ and tonic pain ${ }^{48}$ have also been described. These inconsistencies most probably reflect differences in experimental design: for example, all the groups except the Hammersmith/Manchester group moved a heat probe at a given temperature from one location to another on the forearm in order to minimise the effect of habituation. This is likely to increase attention to the discriminatory components of the stimulus; this is unlikely to be matched in the control condition. There is little doubt that $S 1$ is involved in the sensory discriminatory components of pain processing (Anderson $\mathrm{J}$, et al; submitted for publication). However, the presence or absence of pain is unlikely to be the main determinant of whether there is an increase in flow in this area.

It would appear, therefore, that the nondiscriminatory components of acute pain are mainly processed in the region of the periaqueductal grey and thalamus, and subsequently in structures which may be the principal subcortical and cortical projections of the medial pain system-the anterior insula cortex, lentiform nucleus, mid- and perigenual cingulate cortices, inferior parietal cortex, and prefrontal cortex (dorsolateral prefrontal (DLPF) areas 9, 10, and 46).

The cingulate cortex is involved in a number of integrated tasks including cognition, affect, and response selection. ${ }^{29}$ Although injection of local anaesthetic into the cingulum bundle induces analgesia in animals, ${ }^{56}$ removal or deafferentation of the anterior cingulate cortex in patients with severe intractable pain did not abolish the pain: rather, the patients reported that the pain was no longer bothersome. ${ }^{37}$ This suggests that the cingulate cortex is not necessary for the registration of pain, but may be responsible for the attentional and affective responses to pain. Cognitive tasks which make severe attentional demands, such as the Stroop conflict task, commonly activate the right anterior cingulate cortex. The Stroop task consists of asking subjects to call out the ink colour of colour words which are incongruent with their colour (such as the word 'red' written in green ink).

To what extent the anterior cingulate responses to pain involve the 'attentional network' is not established. A series of experiments to determine whether similar areas of cingulate cortex are activated by an attentionally demanding task (Stroop) and pain in the same individuals suggested that both pain and attention activate structures within the mid cingulate cortex, but that only pain activates the more anterior perigenual cingulate cortex. ${ }^{29} 50$ However, the same networks within the midcingulate are not involved in both tasks, and the anterior cingulate responses to pain appear to be more than just attentional processing. There is some evidence that the perigenual cingulate is involved in affective, vocalisation, and autonomic responses to pain. ${ }^{32}$ It is possible that the anterior cingulate responses comprise a linked attentional and affective network concerned with response selection, the mid-cingulate response being concerned with motor response selection, and the passive attentional and affective components of the response being processed more anteriorly. In support of this suggestion, there are important connections betwen mid-cingulate cortex and the DLPF cortex which has been associated with the generation of willed actions and response inhibition. ${ }^{57}$

The inferior parietal cortical involvement in the pain response may be concerned with the posterior attentional system that Posner and Petersen suggested is involved in directed spatial attention. ${ }^{58}$ However, with its connections to the posterior cingulate cortex, where substantial reductions in flow in response to pain have been recorded, it is more likely that this represents a nociceptive visual orientation response, as has been demonstrated in animals.

\section{Altered functional anatomy during acute} and chronic inflammatory pain

Jones et al studied the cerebral responses to the non-discriminatory components of phasic 
thermal pain (as described earlier) in a group of six patients with active rheumatoid arthritis (RA) according to American Rheumatism Association criteria and compared their responses in the presence of arthritic pain with a group of pain free normal volunteers. ${ }^{59}$ All patients with RA had involvement of the metacarpophalangeal joints, but none of the patients had active synovitis under the area of skin stimulated. Sensory testing and thermal threshold measurements were normal. The same group (Jones AKP, et al; submitted for publication) found that patients with RA demonstrated significantly reduced frontal (BA 10) and anterior cingulate (BA 24 and 32) responses to experimental pain compared with controls (fig 2 ), in addition to reduced responses in the anterior insula and inferior parietal cortex. In the patients with $\mathrm{RA}$, there was a generally damped cortical and subcortical response to phasic thermal pain (fig 3).

These differences are likely to represent an important cortical adaptation in the presence of continuing inflammatory pain. It is not possible to determine the level at which these cortical responses are modified by ascending inputs. The anterior insula receives projections from nociceptive specific components of the ventromedial nucleus of the thalamus. ${ }^{19}$ Both the anterior cingulate and frontal cortices are innervated by the parafascicular and medial thalamic group of nuclei. The cingulate cortex is also implicated in avoidance conditioning ${ }^{60}$ attention, ${ }^{61}$ mood, ${ }^{62}$ and willed actions. Study design and spatial resolution have not enabled the various components of the anterior cingulate response to be discriminated. However, it is likely that the reduced responses to phasic pain may be related to enhanced coping and attentional strategies ${ }^{63}$ related to altered response selection. Support for this concept comes from observations in patients with atypical facial pain who have no identifiable source of nociceptive input, but poor coping strategies, with frequent depression. They show enhanced anterior cingulate responses to standardised experimental heat pain. ${ }^{64}$ However, patients with acute pain attributable to a definite nociceptive input from the face four hours after molar extraction have reduced anterior cingulate and prefrontal cortical responses, with intact thalamic and lentiform nucleus responses to a standardised thermal pain stimulus (Derbyshire SWG, et al; submitted for publication). These different patterns of cortical response suggest that adaptation may be mainly at the level of the thalamocortical projections of the medial thalamic nuclear group.

There are extensive projections from the dorsolateral prefrontal cortex, including areas 9,46 , and $10^{65}$ to the anterior cingulate cortex in primates. Reduced response in the DLPF cortex has also been found in patients suffering atypical facial pain ${ }^{66}$ and dental pain, and depression. ${ }^{67}$ The DLPF cortex has been implicated in the normal supervision of attentional processes, ${ }^{68}$ depressed mood (areas 9,46 ) and willed actions (areas 46, 10). The decreased flow seen in the patients studied may be related

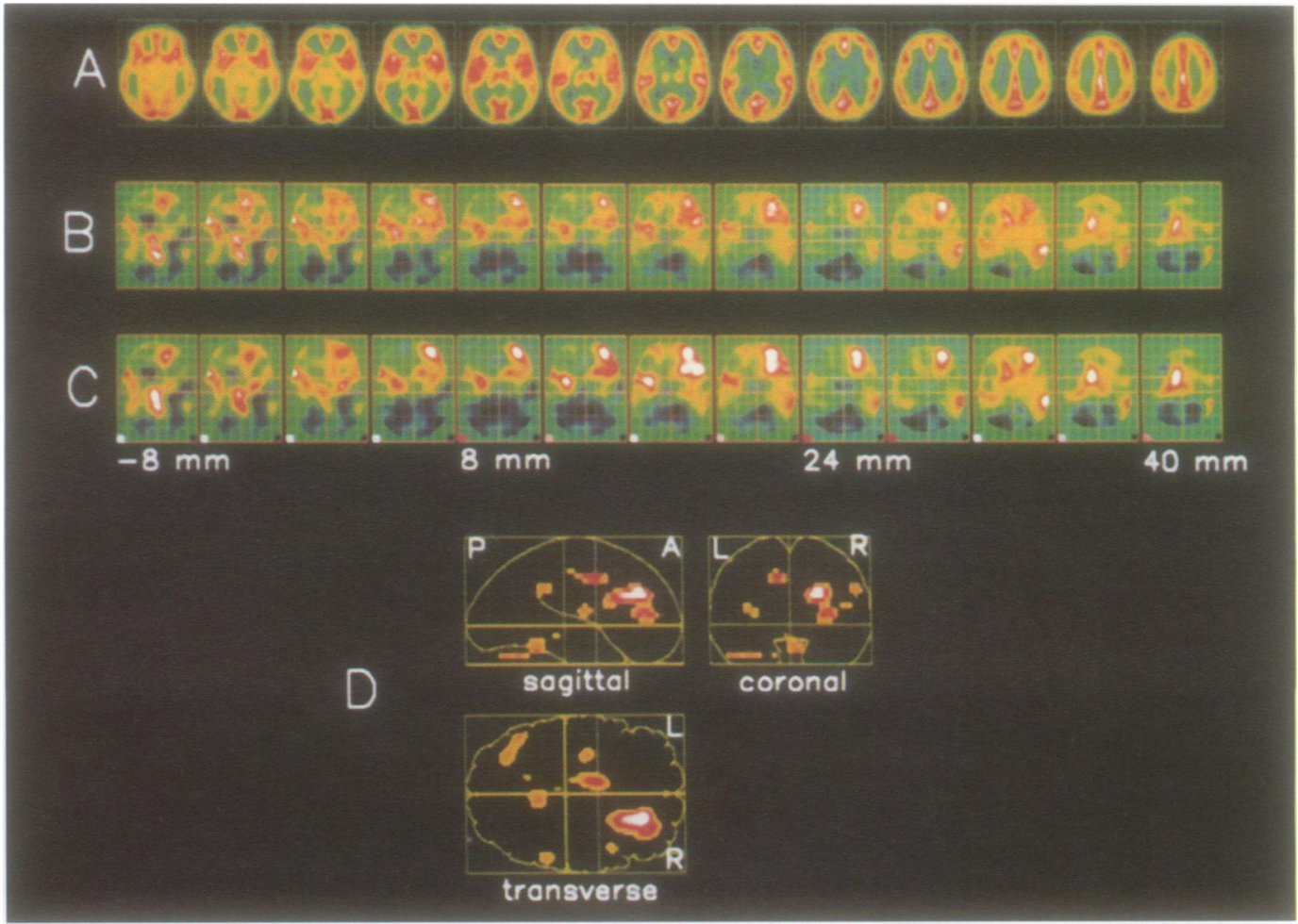

Figure 2 Pain versus heat-female controls. Data averaged from a group of six women. At the top are transverse images of the brain after stereotaxic normalisation, with the distances from the anterior commissure-posterior commissure plane indicated. A: Anatomical features obtained by averaging all blood flow scans from the six women. B: Arithmetical difference between adjusted mean blood flows for painful hot and non-painful hot stimuli. $C$ : Statistical parametric map $\mathrm{t}$ values derived from the formal pixel by pixel comparison of the adjusted mean blood flows and variances for each of the two values derived from the formal pixel by pixel comparison of the adjusted mean blood flows and variances for each
conditions. The colour scale is arbitrary; threshold significance is indicated by the lower lefi pixel for each plane.

D: Orthogonal projections of the statistical comparison at $p<0.001$ ( $\mathrm{Z}$ threshold 3.09). The areas showing significant increases in blood flow are within the region of periaqueductal grey, lentiform nucleus, insula, frontal areas 32 and 9 parietal area 40, and anterior cingulate cortex. 


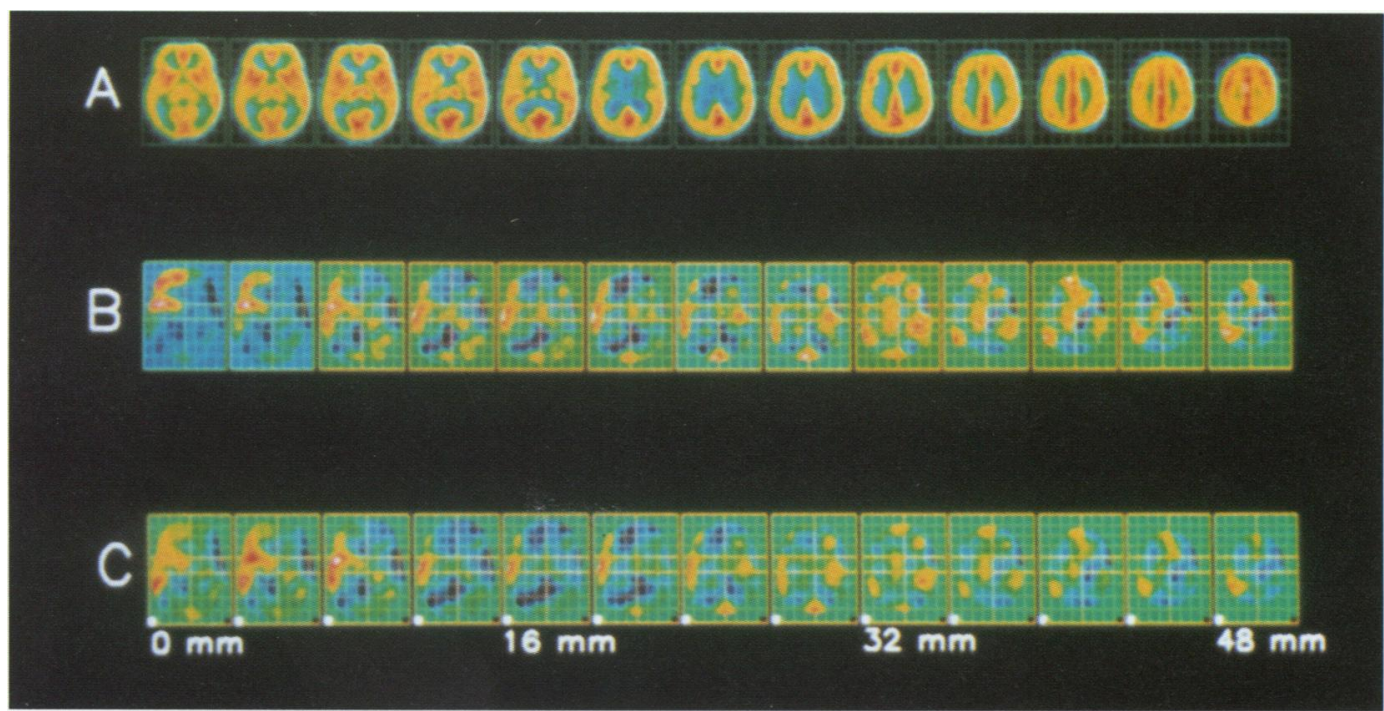

Figure 3 Pain versus heat-rheumatoid arthritis. Data averaged from a group of six $R A$ patients. At the top are transverse images of the brain after stereotaxic normalisation, with the distances from the anterior commissure-posterior commissure plane indicated. A: Anatomical features obtained by averaging all blood flow scans from the six patients. B: Arithmetical difference between adjusted mean blood flows for painful hot and non-painful hot stimuli. C: Statistical parametric map $\mathrm{t}$ values derived from the formal pixel by pixel comparison of the adjusted mean blood flows and variances for each of the two conditions. The colour scale is arbitrary; threshold significance is indicated by the lower left pixel for each plane. No area shows significant increase in regional cerebral blood flow.

to the collapse of this supervisory function and subsequent automatic attempts to recruit coping strategies or catastrophising schemes, depending on the beliefs and expectations of the patients. In this context, it is interesting that Hsieh et al have shown that prefrontal responses to pain are critically dependent on the psychological state of the subject: if the subject is preconditioned by a painful stimulus and knows when to expect the painful stimulus, there are reduced rather than increased prefrontal (ventromedial) responses. ${ }^{69}$ Further sequential studies are in progress to resolve these issues. Recently developed surface electrophysiological mapping techniques allow frontal responses to pain to be studied in detail. The late vertex pain evoked potential, which probably corresponds to cingulate response, has been shown to be dependent on pain intensity. ${ }^{70}$ Sequential studies in patients with inflammatory pain using these techniques may well be the most sensitive and oost effective way of correlating behaviour with physiological events in the forebrain.

A striking reduction in response is thus clearly demonstrated in frontal and anterior cingulate cortical responses during inflammatory pain and is distinct from previously reported increased cingulate responses in patients with psychogenically maintained pain, suggesting different cortical substrates of these two types of pain experience. At present it is difficult to explain these observations in terms of any mechanisms that have been described in animal studies because no equivalent experiment has been done. Studies in rodents with induced hindpaw inflammatory arthritis have demonstrated neurones with expanded receptive fields and prolonged afterdischarges to touch in $S 1$, but not in the ventrobasal complex of the thalamus. ${ }^{66}$ This increase in the receptive field of a unit was correlated with its increased spontaneous activity. The same group also found larger numbers of units with prolonged afterdischarges in response to movement or light pressure of the inflamed joint in the ventrobasal complex of the thalamus and $S 1$ up to one day after induced inflammation. These interesting effects were substantially blocked by both morphine and non-steroidal analgesics. This group made a further interesting observation that the total proportion of units excited by non-joint inputs (brush and pinch) was considerably decreased after induction of arthritis, ${ }^{67}$ and this effect was most marked for the cortex. However, on the current evidence it is not clear that these responses are nociceptive specific, as control non-nociceptive stimulation was not performed. It is possible that joint movement may comprise considerable non-nociceptive proprioceptive stimulation and pinch will also contain considerable touch stimulation. It is also a concern that noxious pressure on the joint may frequently not elicit any thalamic or primary somatosensory cortical response (Guilbaud G; personal communication). The possibility therefore exists that, whereas these observations are undoubtedly related to the presence of inflammatory pain, they may not be related to pain processing but to altered proprioceptive processing. It seems likely that the primary somatosensory cortex is mainly involved in the localisation of pain in both time and spacethat is, the sensory discriminative components of pain. Adaptive motor responses during inflammatory arthritis will certainly require more precise proprioceptive processing. An expansion of receptive fields to nonnociceptive stimuli such as touch within the cortex would be consistent with this process. Human studies are necessary to validate this hypothesis.

The involvement of the anterior cingulate and frontal cortices in addition to medial thalamic nuclei in continuing inflammatory pain in animals has been recently established in 
freely moving rodents using 2-deoxyglucose autoradiography. This was performed at different stages of the development of the acute inflammation in response to injection of formalin into a single paw of rats, and demonstrated the sequence of metabolic changes resulting from increased neuronal activity in the spinal cord, brain stem $^{71}$ and telencephalic structures (Porro CA, et al; personal communication). In addition to significant activation of bilateral medullary, pontine reticular formation, and superior colliculi, several higher structures were activated bilaterally, including medial and lateral posterior thalamic nuclei, parietal, anterior cingulate, anterior insula, and S1 and S2 cortices. Interestingly, the medial thalamic nuclei were activated throughout the period of study (two, 30, and 60 minutes). Frontal, orbitofrontal, S1, S2, anterior cingulate cortex, and portions of the basal ganglia were activated during the second phase of the response. These patterns may obviously be influenced by motor response, but they are very different from the pattern of activation during repetitive motor response (Porro CA, et al; personal communication).

\section{Pharmacology}

The neuropharmacological basis of the differences of cortical responses to pain are not known, but indirect evidence implicates the endogenous opioid system. Substantial changes in opioid receptor binding during inflammatory

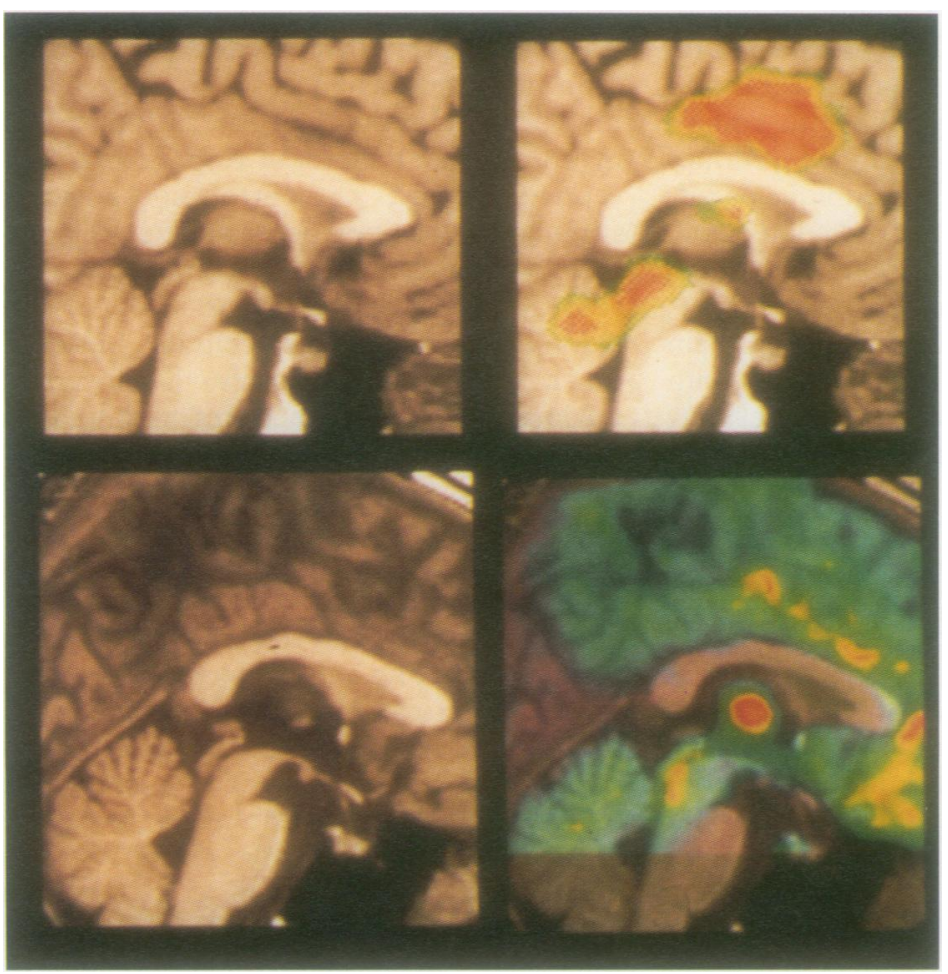

Figure 4 Data from two single subjects in which the magnetic resonance images shown on the left, have been coregistered and superimposed with the positron emission tomography images on the right. A medial slice through the left hemisphere is depicted. TOP: Cerebral response to a phasic heat pain stimulus compared with a non-painful heat stimulus, showing increased regional cerebral blood flow in the anterior cingulate cortex, thalamus, and periaqueductal grey matter in the brain stem. BELOW. Distribution of opiate receptors imaged with ${ }^{11} C$-diprenorphine (binding to $\mu, \delta$, $\kappa$ subtypes), displayed as a parametric map of volume of distribution of receptor binding. The concentration of opiate receptors is maximal in the thalamus and is also high in the anterior parts of the cingulate cortex and in the periaqueductal grey. pain have been demonstrated in patients with arthritis, ${ }^{72}$ consistent with increased cortical opioid peptide release demonstrated in animals with arthritis. ${ }^{73}$ To date, the principal cerebral neuropharmacological studies on inflammatory pain in man have been performed using PET scanning. This technique was used to quantitate accurately the regional cerebral kinetics of tracer quantities of carbon-11 labelled diprenorphine for 90 minutes after intravenous injection. This tracer binds to available $\mu, \delta$, and $\kappa$ opioid binding sites; the radioactivity retained is directly proportional to the concentration of available opioid binding sites. ${ }^{74}$ Binding is quantitated by fitting the regional kinetic data obtained with the PET camera to the continuously sampled arterial plasma radioactivity after correcting for metabolism of the tracer. The error on the fitted values for regional ${ }^{11} \mathrm{C}$-diprenorphine binding has been minimised in these studies by injecting a saturating dose of the opiate antagonist, naloxone, 30 minutes after the injection of tracer. At that point in the fitting routine, the assumption can be made that there is no further binding of ${ }^{11} \mathrm{C}$-diprenorphine to specific binding sites, reducing the number of variables by one. The kinetics of each of a number of brain regions are fitted to derive quantitative values of in vivo ${ }^{11} \mathrm{C}$-diprenorphine binding. These measurements are made in each subject on one occasion while they are in considerable pain and on a second after substantial pain relief. The changes in ${ }^{11} \mathrm{C}$-diprenorphine binding are expressed as a volume of distribution of specific binding and are assumed to reflect changes in occupancy of opioid receptors by endogenous opioid peptides, reducing the chances of trace quantitites of ${ }^{11} \mathrm{C}$-diprenorphine associating with those receptors.

A group of patients with active rheumatoid arthritis was studied during a period of active inflammation and, subsequently, substantially pain free as a result of either natural remission of their disease or intra-articular injection of locally injected and retained steroid. Pain free patients and patients in pain were also compared with non-age-matched controls. All patients who were studied twice showed a substantial reduction in the Ritchie index, erythrocyte sedimentation rate, $C$ reactive protein concentration, or all three parameters, at the time of the second study, suggesting a considerable reduction in inflammation. Visual analogue scale pain scores (both affective and sensory components) for the 24 hours before the scan were reduced by $100 \%$ in three patients and by $62 \%$ in one patient at the time of the second scan. There was very little change in pain threshold, though there was an increase in pain tolerance consistent with the reduction in arthritic pain.

Within group analysis of the four patients scanned in two pain states showed substantial changes in ${ }^{11} \mathrm{C}$-diprenorphine binding in most brain regions analysed except for inferior temporal, inferior and mid-occipital cortices, the primary visual cortex (cuneus), and posterior putamen. ${ }^{72}$ Significant decreases in binding were observed in the superior and 
inferior frontal cortices, straight gyrus, anterior and posterior cingulate, superior, and midtemporal cortices. None of the changes seen in the subcortical structures achieved significance. In this particular study, no distinction can be made between the effects of inflammation and pain. It is therefore possible that all the changes observed may have been attributable to the inflammation alone. However, a similar pattern of changes in opiate receptor binding has been observed in non-inflammatory neurogenic pain, suggesting that persistent pain is probably an important component of the stimulus necessary to elicit such responses. Another possibility is that this may be a non-specific response to chronic stress. Severe physiological stress induced in rodents by swimming in ice cold water caused a substantial displacement of in vivo ${ }^{3} \mathrm{H}$-diprenorphine binding in a number of subcortical structures, including the medial thalamus, amygdala, and periaqueductal grey. ${ }^{75}$ Human stress responses may deplete serotonin reserves and disrupt 5-hydroxytryptamine mediated descending analgesia, ${ }^{76}$ or directly interfere with opiate responses in the cingulate cortex itself. While it is unlikely that the observations in patients with inflammatory pain can be explained purely on the basis of a stress response, many have suggested that the pain of arthritis is mediated by the effects of stress in certain personalities. ${ }^{77} 78$ Others have commented that psychological characteristics and attitudes to illness are more important predictors of disability and pain than the severity and activity of the arthritis. ${ }^{6379}$

Figure 4 illustrates the differential binding of opiates in the brain structures relating to the medial pain system, and indicates that the opioid binding closely mirrors the medial response to pain seen largely in the anterior cingulate cortex. Neurones in the anterior cingulate cortex show a plasticity that is not evident within the highly somatotopic structure of S1. ${ }^{80-82}$ This may explain the wide heterogeneity of cingulate function as discussed earlier, and may also explain why cognitive coping strategies for dealing with cold pressor induced pain have been shown to be less effective after administration of naloxone. ${ }^{83}$ Strategies for dealing with the pain may become less accessible.

There is now evidence that the later $\beta$-endorphinergic components of the endogenous opiate response during inflammation may be mainly modifying behaviour at a supraspinal level. The intraventricular injection of antibody to $\beta$-endorphin in rats appears to cause a reduced pain response correlated with reduced brain activity at a supraspinal level only (Porro CA, et al; personal communication). The authors tentatively suggest that there may be a sequence of opioid mediated events in the brain, as follows. The early release of endogenous opiates may be associated with reinforcement of suppression of motor responses, facilitating neuronal plasticity within the frontal and cingulate cortices, and allowing greater development of psychological coping strategies. Prolonged opioid activation may, via descending afferents from these cortical areas to medial thalamic and periaqueductal grey in the brain stem, result in a resetting of thresholds for ascending nociceptive inputs to these higher centres. A series of prospective and longitudinal studies are now planned to test these hypotheses.

1 Melack R, Wall P. The challenge of pain. Harmondsworth: Penguin Books, 1991

2 Boring E G. Sensation and perception in the history of experimental psychology. New York: Appleton-CenturyCrofts, 1942.

3 Bottini G, Paulescu E, Sterzi R, et al. Modulation of conscious experience by peripheral sensory stimuli. Nature 1995; 376: 778-81.

4 Nathan P W. Pain. Br Med Bull 1977; 33: 149-56.

5 Brown-Sequard J. Course of lectures on the physiology and pathology of the central nervous system. Philadelphia: Lippincott, 1860.

6 Sherrington C S, Laslett E E. On the anatomical course of reflex connections in the spinal cord. F Physiol (Lond) 1903; 29: 58-96

7 Vierck C J, Luck M M. Loss and recovery of reactivity of noxious stimuli in monkeys with primary spinothalamic cordotomies, followed by secondary and tertiary lesions of other cord sectors. Brain 1979; 102: 233-48.

8 Meheler W R, Feferman M E, Mauta W J H. Ascending axon degeneration following antero-lateral cordotomy. An experimental study in monkey. Brain 1960; 83: 718-50.

9 Apkarian A V, Hodge C J. Primate spinothalamic pathways: II. The cells of origin of the dorsolateral and ventral spinothalamic pathways. f Comp Neurol 1989; 288: 474-92.

10 Apkarian A V, Hodge C J. Primate spinothalamic pathways: I. A quantitative study of the cells of origin of the spinothalamic pathway. $f$ Comp Neurol 1989; 288: 447-3

11 Apkarian A V, Hodge C J. Primate spinothalamic pathways: III. Thalamic terminations of the dorsolateral and ventral spinothalamic pathways. f Comp Neurol 1989; 288: 493-511.

12 Boivie J. An anatomical reinvestigation of the termination of the spinothalamic tract in the monkey. $\mathcal{F}$ Comp Neurol 1979; 186: 343-70.

13 Shi T, Stevens R T, Tessier J, Apkarian A V. Spinothalamic inputs nonpreferentially innervate the superficial and deep cortical layers of S1. Neurosci Lett 1993; 160: 209-13.

14 Stevens R T, London S M, Apkarian A V. Spinothalamic projections to the secondary somatosensory cortex (SII) in squirrel monkey. Brain Res 1993; 631: 241-6.

15 Kenshalo D R, Isense O. Responses of the primate SI cortical neurons to noxious stimuli. F Neurophysiol 1983; 50: $1479-96$.

16 Strick P L. Light microscopic analysis of the cortical projection of the thalamic ventrolateral nucleus in the cat. Brain Res 1973; 55: 1-24.

17 Lin C S, Merzenich M M, Sur M, Kaas J H. Connections of area $3 \mathrm{~b}$ and 1 of the parietal somatosensory tip with
the ventroposterior nucleus in the owl monkey (Actus triviratus). $\mathcal{F}$ Comp Neurol 1979; 185: 355-72.

18 Burton $\mathrm{H}$, Jones $\mathrm{E} \mathrm{G}$. The posterior thalamic region and its cortical projection in new world monkeys. $\mathcal{F}$ Comp Neurol 1976; 168: 249-301.

19 Craig A D, Bushnell M C, Zhang E T, Blomquist A. A thalamic nucleus specific for pain and temperature sensation. Nature 1994; 372: 770-3.

20 Kerr F W L. The ventral spinothalamic tract and other ascending systems of the ventral funiculus of the spinal cord. 7 Comp Neurol 1975; 159: 335-55.

21 Kerr F W L, Lippman H H. The primate spinothalamic tract as demonstrated by anterolateral cordotomy and tract as demonstrated by anterolateral cordotomy and

22 Bing Z, Villanueva L, Le Bars D. Ascending pathways in the spinal cord involved in the activation of subnucleus reticularis dorsalis neurons in the medulla of the rat. $\mathcal{f}$ Neurophysiol 1990; 63: 424-37.

23 Bernard J F, Villanueva L, Carroue J, Le Bars D. Efferent projections from the subnucleus reticularis dorsalis (SRD): a Phaseolus vulgaris leucoagglutinin study in the rat. Neurosci Lett 1990; 116: 257-62.

24 Macchi $G$, Bentivoglio $M$. The thalamic intralaminar nuclei and the cerebral cortex. In: Jones E G, Peters A, eds. and the cerebral cortex. In: Jones E G, Peters A, eds.

25 Vogt B A, Rosene D L, Pandya D N. Thalamic and cortical afferents differentiate anterior from posterior cingulate afferents differentiate anterior from posterior
cortex in the monkey. Science 1979; 204: 205-7.

26 Kievit J, Kuypers R C. Organization of the thalamo-cortical connexions to the frontal lobe in the rhesus monkey. Exp Brain Res 1977; 29: 299-322.

26a Albe-Fessard D, Berkely K J, Kruger L, Ralston H J, Willis W D. Diencephalic mechanisms of pain sensation. Brain Res Rev 1985; 9: 217-96.

27 Vogt B A, Sikes R W, Vogt L J. Anterior cingulate cortex and the medial pain system. In: Vogt B A, Gabriel M, eds. Neurobiology of cingulate cortex and limbic thalamus. Boston, Basel, Berlin: Birkhauser, 1993; 313-44.

28 Sikes R W, Vogt B A. Nociceptive neurons in area $24 \mathrm{~b}$ of rabbit anterior cingulate cortex. $\mathcal{F}$ Neurophysiol 1992; 68: 1720-32. 
29 Devinsky O, Morrell M J, Vogt B A. Contributions of anterior cingulate to behaviour. Brain 1995; 118: 279-306.

30 Tsubokawa T, Katayama Y, Ueno Y, Moriyasu N. Evidence for involvement of the frontal cortex in pain-related
cerebral events in cats: increase in local cerebral blood cerebral events in cats: increase in local cerebral blo
flow by noxious stimuli. Brain Res 1981; 217: 179-85.

31 Chudler E H, Dong W K, Kawakami Y. Cortical nociceptive responses and behavioural correlates in the monkey. Brain Res 1986; 397: 47-60.

32 Melzack R, Casey K L. The sensory, motivational and control determinants of pain. In: Kenshalo D R, ed. The skin senses. Springfield: Thomas, 1968; 423-43.

33 Hosobuchi Y. Subcortical electrical stimulation for the control of intractable pain in humans. Report of 122 cases (1970-1984). F Neurosurg 1986; 64: 543-53.

34 Bowsher D. Termination of the central pain pathway in man: the conscious appreciation of pain. Brain 1957;80: man: the

35 Boukoms A J. Psychosurgery for pain. In: Wall P D, Melzack R, eds. Textbook of pain. Edinburgh, London, Melbourne, New York: Churchill Livingstone, 1989; 868-81

36 Gybels J M, Sweet W H. Pre- and postcentral gyrectomy. In: Gybels J M, Sweet W H, eds. Neurosurgical treatment of persistent pain. Basel, Munich, Paris, London: Karger, $1989 ; 254-6$.

37 Foltz E L, White L E J. Pain relief by frontal cingulotomy. Neurosurgery 1962; 19: 89-100.

38 Tasker R R. Deafferentation. In: Wall P D, Melzack R, eds. Texbook of pain. Edinburgh, London, Melbourne, New York: Churchill Livingstone, 1984; 119-32.

39 Penfield W, Boldrey E. Somatic motor and sensory representation in the cerebral cortex of man as studied by electrical stimulation. Brain 1937; 60: 389-443.

40 Woolsey C N, Erickson T C, Gilson W E. Localisation in somatic sensory and motor areas of human cerebral cortex as determined by direct recording of evoked potentials and electrical stimulation. $f$ Neurosurg 1979; 51: 476-506.

41 Sweet W H. Cerebral localisation of pain. In: Thompson RA, Green J R, eds. New perspectives in cerebral localisation. New York: Raven Press, 1982; 205-42.

42 Talairach J, Tournoux P, Bancard J. Chirugie parietale de la douleur. Acta Neurochir 1960; 8: 153-250.

43 Gabriel M, Orona E, Foster K, Lambert R W. Mechanisms and generality of stimulus significance coding in a mammalian model system. Adv Behav Biol 1982; 26: 535-67.

44 Chen A C N. Human brain measures of clinical pain: a review I. Topographic mappings. Pain 1993; 54: 115-32.

45 Lueck C J, Zeki S, Friston K J, et al. The colour centre in the cerebral cortex of man. Nature 1989; 340: 386-9.

46 Raichle M E. Circulatory and metabolic correlates of brain function in normal humans. In: Plum F, Mountcastle V, eds. American Physiological Society Handbook of Physiology: Section 1. The nervous system, Vol V, Part 2. Maryland American Physiological Society, 1987; 643-74

47 Jones A K P, Friston K J, Brown D, Qi L, Frackowiak R S J. Cortical and subcortical localisation of response to pain in man using positron emission tomography. Proc $R$ Soc B 1991; 244: 39-44.

48 Derbyshire S W G, Jones A K P, Vogt B A, Frackowiack R S J. An investigation of central processing with the Stroop task and pain using positron emission tomography. A within subject design. $f$ Cereb Blood Flow Metab 1995; 15(suppl 1): S860.

49 Casey K L, Monoshima S, Berger K L, Koeppe R A, Morrow T J, Frey K A. Positron emission tomographic analysis of cerebral structures activated specifically by analysis of cerebral structures activated specifically by repetitive

50 Talbot J D, Marrett S, Evans A C, Meyer E, Bushnell M C, Duncan $G \mathrm{H}$. Multiple representations of pain in human cerebral cortex. Science 1991; 251: 1355-8.

51 Di Piero V, Ferracuti S, Sabatini, Pantano P, Crucci G, Lenzi G L. A cerebral blood flow study on tonic pain activation in man. Pain 1994; 56: 167-73.

52 Vogt B A, Derbyshire S, Jones A K P. Pain processing in four regions of the human cingulate cortex localised with coregistered PET and MR imaging. Eur $₹$ Neurosci 1996. In press.

53 Hsieh J C, Belfrage $M$, Stone-Elander S, Hansson P, Ingvar $M$. Central representation of chronic ongoing neuropathic pain studied by positron emission tomography. Pain 1995; 63: 225-36.

54 Jones A K P, Hughes D G, Robinson L, Sykes J R, Derbyshire S W G, Chen A C N. Functional magnetic resonance imaging at 1 Tesla in the study of pain. Proceedings of the Society of Magnetic Resonance 3rd Scientific Meeting, Nice. Berkeley: Society of Magnetic Resonance, 1995; 149

55 Apkarian V A, Stea R A, Manglos S H, Szevernyi N M, King $R$ B, Thomas F D. Persistent pain inhibits contralateral somatosensory cortical activity in humans. Neurosci Lett 1992; 140: 141-7.

56 Vaccarino A L, Melzack R. Analgesia produced by injection of lidocaine into the anterior cingulum bundle of the rat. Pain 1989; 39: 213-9.

57 Goldman-Rakic P S. Circuitry of the prefrontal cortex and the regulation of behaviour by representational memory.
In: Plum F, Mountcastle V, eds. American Physiology Society Handbook of Physiology. Section 1. The nervous
system, part I, Chapter 9. Maryland: American system, part I, Chapter 9. Maryland: American
Physiological Society, $1987 ; 373-417$. 58 Posner M I, Petersen S E. The attentional system of the human brain. Ann Rev Neurosci 1990; 13: 25-42.

59 Jones A K P, Derbyshire S W G, Pearce S. Reduced central responses to pain in patients with rheumatoid arthritis. $\mathrm{Br}$ f Rheumatol 1993; 32(suppl 2): 20

60 Gabriel M, Sparenborg S P, Kubota Y. Anterior and medial thalamic lesions, discriminitative avoidance learning, and cingulate cortical neuronal activity in rabbits. Exp Brain Res 1989; 76: 441-57.

61 Pardo J V, Fox P T, Raichle M E. Localisation of a human system for sustained attention by positron emission system for sustained attention by

62 Bench C J, Dolan R J, Friston K J, Brown R G, Scott L C Frackowiak R S J. The anatomy of melancholia-focal abnormalities of cerebral blood flow in major depression and the cognitive impairment of depression. Psychol Med 1991; 22: 1-9.

63 Keefe F J, Caldwell D S, Martinez S, Nunley J, Beckham J, Williams D A. Analyzing pain in rheumatoid arthritis patients. Pain coping strategies in patients who have had knee replacement surgery. Pain 1991; 46: 153-60.

64 Derbyshire S W G, Jones A K P, Devani P, et al. Cerebral responses to pain in patients with atypical facial pain measured by positron emission tomography. 7 Neurol Neurosurg Psychiatr 1994; 57: 1166-72.

65 Barbas H, Pandya D N. Architecture and intrinsic connections of the prefrontal cortex in the rhesus monkey. f Comp Neurol 1989; 286: 353-75.

66 Vin-Christian K, Benoist J M, Gautron M, Levante A, Guilbaud G. Further evidence for the involvement of SmI cortical neurons in nociception: modifications of their responsiveness over the early stage of a carrageenininduced inflammation in the rat. Somatosens Motor Res 1992; 9: 245-61.

67 Guilbaud G, Benoist J M. Central transmission of somatosensory inputs in the thalamic ventrobasal complex and somatosensory cortex in rat models of clinical pain. In: Boivie J, Hansson P, Lindblom U, eds. Touch, temperature and pain in health and disease: mechanisms and assessments. and pain in health and disease: mechan

68 Posner M I, Rothbart M K. Attentional mechanisms and conscious experience. In: Milner D, Rugg M, eds. The neurophysiology of consciousness. London: Academic Press, 1991; 91-111.

$69 \mathrm{Hsieh}$ J C. Anticipatory coping for pain revealed by positron emission tomography. In: Central processing of pain: functional brain imaging studies with PET. [Thesis] Stockholm, 1995. ISBN: 91-628-1722-1.

70 Chen A C N, Chapman C R, Harkins S W. Brain evoked potentials are functional correlates of pain in man. Pain 1979; 6: 365-74.

71 Porro C A, Cavuzzuti M, Spatial and temporal aspects of spinal cord and brainstem activation in the formalin pain model. Prog Neurobiol 1993; 41: 565-607.

72 Jones A K P, Cunningham V J, Ha-Kawa S, Fujiwara T, Luthra $S \mathrm{~K}$, Jones T. Changes in central opioid receptor binding in relation to inflammation and pain in patients with rheumatoid arthritis. $\mathrm{Br} \mathcal{F}$ Rheumatol 1994; 33: 909-16.

73 Panerai A E, Sacerdote P, Bianchi M, Brini A, Mantergazza P. Brain and spinal cord neuropeptides in adjuvant induced arthritis in rats. Life Sci 1987; 41: 1297-303.

74 Jones A K P, Cunningham V J, Ha-Kawa S, et al. Quantitation of ${ }^{11} \mathrm{C}$-diprenorphine cerebral kinetics in man acquired by PET using presaturation, pulse-chase man acquired by PET using presaturation, pulse-chase and tracer

75 Seeger T F, Sforzo G A, Pert C B, Pert A. In vivo autoradiography: Visualisation of stress-induced changes in opiate receptor occupancy in the rat brain. Brain Res 1984; 305: 303-11.

76 Melzack R, Stotler W A, Livingstone W K. Effects of discrete brainstem lesions in cats on perception of noxious stimulation. $\mathcal{7}$ Neurophysiol 1958; 21: 353-67.

77 Hawley D J, Wolfe F. Anxiety and depression patients with rheumatoid arthritis: a prospective study of 400 patients. f Rheumatol 1988; 15: 932-41.

78 Parker J, Frank R, Beck N, et al. Pain in rheumatoid arthritis: relationship to demographic, medical and arthritis: relationship to demographic, medical

79 McFarlane A C, Brooks P M. An analysis of the relationship between psychological morbidity and disease activity in rheumatoid arthritis. F Rheumatol 1988; 15: 926-31.

$80 \mathrm{Gabriel} M$. Functions of anterior and posterior cingulate cortex during avoidance learning in rabbits. In: Uylings H, Van Eden C, De Bruin J, Corner M, Feenstra M, eds. Progress in brain

81 Gabriel M, Foster K, Orona E. Interaction of laminae of the cingulate cortex with the anteroventral thalamus during behavioural learning. Science 1980; 208: 1050-2.

82 Gabriel M, Foster K, Orona E, Saltwick S E, Stanton M. Neuronal activity of cingulate cortex, anteroventral thalamus, and hippocampal formation in discriminative conditioning. Progr Psychobiol Physiol Psychol 1980; 9: $125-231$.

83 Bandura A, Cioffi D, Taylor C B, Brouillard M E. Perceived self-efficacy in coping with cognitive stressors and opioid activation. F Pers Soc Psychol 1988; 55: 479-88. 